for about $20 \mathrm{~h}$. Afterwards, stirring the slurry in a vacuum desiccator helped to eliminate any trapped air. Then the slurry passed to a cylindrical container specially designed for this experiment. This container had one end immersed in ethanol cooled at $-50^{\circ} \mathrm{C}$. They fabricated this section of the container with a metallic material of high conductivity. For the sides of the container, they used a resin. The other end of the container remained open to the air allowing growth of frozen ice in a vertical direction. Drying of the ice thus formed took one day in conditions of reduced pressure. The sintering conditions of each sample were $1400^{\circ} \mathrm{C}$ and $1550^{\circ} \mathrm{C}$, both for $2 \mathrm{~h}$.

Two slurries were prepared at different concentrations. Measurements of their rheological properties revealed a uniform distribution of the alumina powders. The freeze-dried samples showed large pores aligned with the growth direction. After freeze-drying, the volume change measured was almost negligible. Pore-size distributions measured after sintering have two peaks at $0.1 \mu \mathrm{m}$ and $10 \mu \mathrm{m}$ for the samples sintered at $1400^{\circ} \mathrm{C}$. On the other hand, the pore-size distributions of the samples sintered at $1550^{\circ} \mathrm{C}$ only have one peak at around $10 \mu \mathrm{m}$. Inspection of these samples using a scanning electron microscope allowed the detection of a dendritic structure inside the large pores for all samples. In the case of the sample sintered at $1400^{\circ} \mathrm{C}$, inside the dendritic structures there were small pores. These pores correspond to the peak at $0.1 \mu \mathrm{m}$ observed in the pore-size distribution. It was also possible to detect a decrease in the pore size with increasing concentration of the slurry. Furthermore, the researchers' current results indicate that by changing the freezing temperature, control of the macroscopic pore size is possible. Since the pores appear before sintering, this method is applicable to any ceramic. Recently, the researchers obtained porous silicon nitride after application of this technique.

SIARI S. SOSA

\section{Electrostatic Attraction Enables Precise Control in Thin-Film Assembly}

A research group at the University of Massachusetts-Lowell has prepared a thin film consisting of oppositely charged alternating layers of organic laser dye molecules intercalated into an aluminosilicate lattice and polycation spacer layers. The components of the film were sequentially deposited onto a glass substrate and allowed to assemble by electrostatic attraction. This method allowed for precise control of film thickness and dye molecule orientation in the film. The dye molecules in the assembly were more thermally stable than free dye and exhibited a high degree of order.

As reported in the March issue of Chemistry of Materials, the dye molecules (coumarin 1 or 7-diethylamino-4-methyl coumarin) were first protonated by hydrochloric acid treatment. A suspension of the aluminosilicate hectorite was then added to the protonated dye solution and ion exchange was allowed to proceed for $24 \mathrm{~h}$. The resulting host/guest composite was separated by centrifugation, washed with water to remove excess coumarin, and suspended in de-ionized water. Composites with coumarin content ranging between $11 \%$ and $252 \%$ of the cation exchange capacity (CEC) of the hectorite were prepared by adjusting the dye/aluminosilicate ratio. The thin-film assembly of the composite was prepared by alternatively depositing layers of positively charged poly(diallyldimethylammonium) chloride and negatively charged composite onto hydrophilized glass slides.

The absorbance and emission intensities of the coumarin were monitored during film assembly and were found to increase linearly with the number of deposition cycles. This is evidence of a controlled buildup of layers, a feature not present in existing aluminosilicate thinfilm preparation methods.

Thermogravimetric analysis of the thin films showed that the loss of coumarin began at $340^{\circ} \mathrm{C}, 190^{\circ}$ higher than that observed for the free dye. The researchers attributed this improved thermal stability to the restricted mobility of the dye molecules in the hectorite lattice. The orientation of the dye molecules was inferred from the changes in the interlamellar spacing of the hectorite as measured by powder x-ray diffraction. These measurements showed that the interlamellar spacing did not change for composites having coumarin content of up to $100 \%$ CEC. The spacing was found to increase with increasing coumarin content above this value. The researchers propose that at low CEC the guest molecules are oriented flat with respect to the host lattice, while at a higher percentage of CEC they tilt for more efficient packing, the angle of the tilt increasing with increased doping level. This allows for precise control of chromophore orientation in the assembly.

The researchers believe that the ability to control the film thickness and chromophore orientation coupled with the stability and high order of the resultant structure make the preparative method described here "a simple but powerful strategy for preparing a nanocomposite film for photonic applications." They add that the method "may be adaptable to other photofunctional chromophores and fluorophores."

GREGORY KHITROV

\section{Raman Spectroscopy Detects Carotenoid Levels in Human Retina}

A team of researchers at the University of Utah has recently developed a resonant Raman scattering method for the detection of macular carotenoid pigments. In their article in the February 15 issue of Optics Letters, they demonstrate that this approach can be used as a noninvasive technique for the determination of carotenoid levels in the living human retina. Quantitative information could be obtained with laser excitations that were well below the safety limits for macular exposure.

"Age-related macular degeneration is the leading cause of blindness in the elderly in the United States," said Paul S. Bernstein, a professor of ophthalmology at Utah. "The vision loss can be prevented if low levels of carotenoid pigments in the retina are detected at an early stage."

The most commonly used noninvasive technique for quantification of carotenoid pigment levels is a subjective psychophysical flicker photometric test that involves color matching of two light beams aimed at the fovea and the perifoveal area. This method is not only time intensive, but also requires the attention of the subject and good visual acuity. Bernstein said that "this limits the usefulness for assessing macular pigment levels in the elderly population that is most at risk." In collaboration with colleagues in the Department of Physics, he developed a fast and objective method for pigment level measurements based on resonant Raman scattering.

The two carotenoid pigments that are concentrated in the human retina, lutein and zeaxanthin, are polyene-like molecules with a conjugated $\pi$-bonding system along the carbon backbone.

"The molecules exhibit extremely low luminescence efficiency and no fluorescence due to nonradiative decay from the excited state to a second excited state, from which electronic emission to the ground state is symmetry forbidden," said Werner Gellermann, a professor in the Physics Department. "This allows us to explore the resonant Raman scattering response without having to worry about potentially masking fluorescence signals from the carotenoids."

Preliminary experiments were conducted in a $180^{\circ}$ backscattering geometry using an optically correct model eye to which 
tissue papers with well-defined lutein concentrations were attached. Strong and clearly resolved Raman signals were measured with the intensity varying linearly with lutein concentration. Further experiments were carried out on the healthy eye of a volunteer. In these measurements the laser power was $300 \mu \mathrm{W}$, the exposure time $1 \mathrm{~s}$, and the spot diameter on the macula maintained at about $1 \mathrm{~mm}$. This corresponds to a dose of $\sim 38 \mathrm{~mJ} / \mathrm{cm}^{2}$, a value which is about 400 times lower than the ANSI maximum permissible safe dose of $15.5 \mathrm{~J} / \mathrm{cm}^{2}$.

"Raman signals are typically of very weak intensity and therefore we usually need to use relatively powerful lasers in combination with sophisticated light collection and analysis instrumentation," said Gellermann. "As physicists, we're trained to not to stare into lasers. But, when our colleagues in ophthalmology pointed out the strong need to measure macular pigments in a noninvasive and objective way, we decided to take this unique approach and it has worked out very well."

CORA LIND

\section{Silver Selectively Deposited on Ferroelectric Barium Titanate} Domains Enable Creation of Metallic Patterns with Submicron Features

In the February issue of Chemistry of Materials, Jennifer L. Giocondi and Gregory S. Rohrer from the Department of Materials Science and Engineering at Carnegie Mellon University reported that silver had been selectively deposited on some specific ferroelectric $\mathrm{BaTiO}_{3}$ domains during photochemical reduction of aqueous $\mathrm{Ag}^{+}$. This domain decoration-related finding makes it possible to create metallic patterns with submicron features.

In this study, the photochemical reduction was accomplished by exposing the surface of $\mathrm{BaTiO}_{3}$ polycrystals to a $300-\mathrm{W}$ $\mathrm{Hg}$ lamp for $3 \mathrm{~s}$ in the presence of a $0.115 \mathrm{M}$ aqueous $\mathrm{AgNO}_{3}$ solution. The sample, after rinsing and drying, was imaged using atomic force microscopy (AFM). By comparing the topographic AFM images of the samples before and after the reduction reaction, silver appeared to be selectively deposited on certain regions of the surface that corresponded to specific domains. Photochemical reduction of silver on $\mathrm{BaTiO}_{3}$ single crystal was conducted and acid-etching techniques were applied by the researchers in order to identify the domains. The AFM images demonstrated that the up domains, where the positive ends of the dipoles point at the surface, etched the fastest and accumulated the most silver. The down domains were the least favored for the silver deposition. The researchers believe that this selective deposition of silver in up domains was not due to the preferential adsorption of $\mathrm{Ag}^{+}$cations before reduction, but to the influence of the static dipolar fields in the domains. The field in up domains pushed electrons to the surface where they could reduce silver, while the field in down domains urged electrons away from the surface where they were unable to react with the silver cations.

According to the researchers, this study provides a potential approach to "creating metallic patterns with submicron features." They said that this highly selective deposition technique would make it possible to "predefine a pattern and then metallize the surface" in the future.

XiAnlong GE

\section{\begin{tabular}{|c|}
\hline Send Letters to the Editor to: \\
Editor, MRS Bulletin \\
Materials Research Society, 506 Keystone Drive \\
Warrendale, PA 15086-7573 USA \\
Fax: 724-779-8313; E-mail: Bulletin@mrs.org \\
Letters must include your full name, institution, address, phone number, and e-mail. \\
\hline
\end{tabular}}

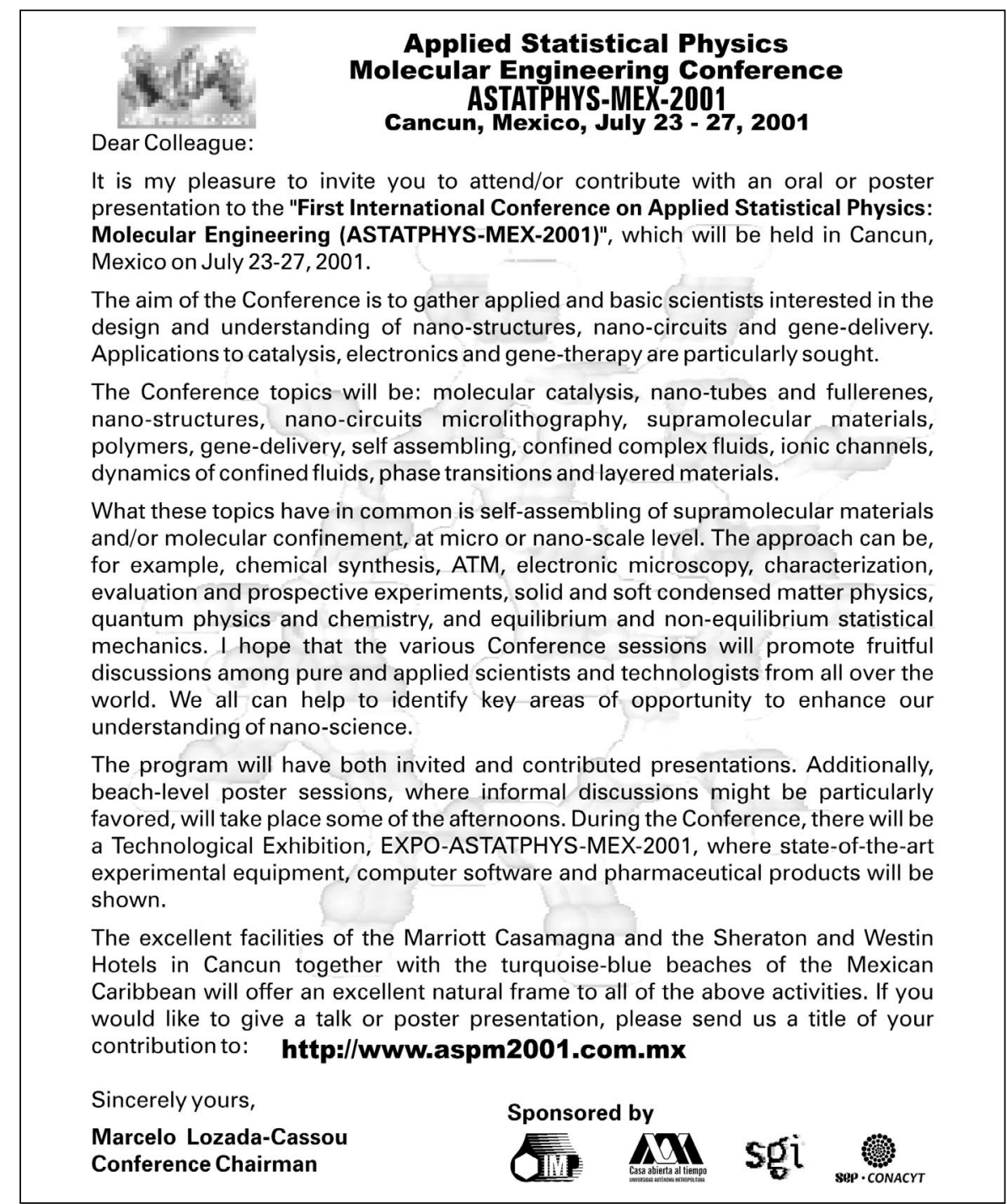

Circle No. 12 on Inside Back Cover 